\title{
Analysis and Simulation of SIRI Model for Dengue Fever Transmission
}

\section{Syafruddin Side ${ }^{1, *}$, Muhammad Isbar Pratama ${ }^{1}$, Nasiah Badwi ${ }^{2}$ and Wahidah Sanusi ${ }^{1}$}

\author{
'Department of Mathematics, Universitas Negeri Makassar, Parangtambung, \\ Makassar, Indonesia \\ 2Department of Geography, Universitas Negeri Makassar, Parangtambung, \\ Makassar, Indonesia
}

\section{Article Type: Article}

Article Citation: Syafruddin Side, Muhammad Isbar Pratama, Nasiah Badwi, Wahidah Sanusi. Analysis and simulation of SIRI model for dengue fever transmission. Indian Journal of Science and Technology. 2020; 13(03), 340-351. Dol: 10.17485/ijst/2020/ v013i03/147852

Received date: September 30, 2019

Accepted date: December 13, 2019

*Author for correspondence: Syafruddin Side syafruddin@unm. ac.id 9 Department of Mathematics, Universitas Negeri Makassar, Parangtambung, Makassar, Indonesia

\begin{abstract}
Objectives: The aim of this study is to obtain SIRI model for dengue fever (DHF) transmission, conduct analysis, and simulation of SIRI model in disease-free and endemic and also to predict the number of DHF cases. Methods/statistical analysis: Dengue fever is caused by a virus carried by the Aedes aegypti and Aedes albopictus mosquitoes, the SIRI model is a modification of the SIR model. Analysis of the SIRI model use the Lyapunov function method, then the data used in the simulation are assuming to show two possible dengue status are disease free and endemic status. The simulation also using the number of dengue case in Makassar city for showing the status of dengue fever transmission in Makassar city. Simulation models using Maple software are to predict the number of dengue cases in the following months. Findings: The results of this study are the SIRI model of the transmission of dengue fever with variables that have recovered can be re-infected with dengue fever, analysis of the SIRI model of dengue transmission provides information that the equation system in the SIRI model which is asymptotically stable, it means that dengue cases always exist at a certain time and certain region. The simulation results of the SIRI model in this study illustrate the number of dengue cases in the following months. While the first simulation found the basic reproduction number is $R_{0}=0.0366 \leq$ 1 this means that dengue transmission is at an alarming stage, but the second simulation finds the basic reproduction number $R_{0}=$ $31.2733>1$, this means that, a person infected with dengue causes eight individuals will be infected with dengue fever, so that it is in the endemic stage, and the last simulation using data of the number of dengue case in Makassar city found $=1$, that means, Makassar city is a free disease case for dengue fever transmission. Application/ improvements: SIRI model for DHF transmission is a mathematical health application. Model analysis guarantees existence, disease-free
\end{abstract}


or endemic status, while simulation results can be used as a reference in DHF prevention.

Keywords: SIRI Model, Dengue Fever, Model Analysis, Simulation, Free Disease, Endemic Dengue.

\section{Introduction}

he World Health Organization [1] states that the number of dengue fever (DHF) cases in the world has increased 30 -fold over the past 50 years, and was named the fastest growing disease in the world and more than half of the world's research results are related quality. In cites, one to one million people were admitted in hospitals to diagnose DHF every year [1]. More than patients were children and 2.5 percent of them eventually died. Data from around the world [1] show that Asia determines the first place in the number of dengue fever patients each year. Meanwhile, starting in 1968 until 2016, Indonesia was the highest country with Dengue Fever in Southeast Asia [1]. Makassar city is one of the districts in South Sulawesi with the highest number of dengue cases. According to the Health Department Makassar City, the number of dengue cases in Makassar in 2016 is 142 cases, 2017 is 248 cases and January to February 2018 is 62 cases [2].

Mathematical modeling to detect and predict the number of dengue cases, namely the SIR and SEIR models have been carried out [3-17]. The models assume that the recovered population is immune, but the current facts show that recovered populations can be re-infected. Based on this, this study will build a SIRI model as a modification of the SIR model that has been carried out by researchers [9-10], then this model is analyzed and then simulated to predict the number of cases in the following years in Makassar city, South Sulawesi which can be a solution to prevent dengue fever.

\section{Material and Method}

\subsection{Study Area and Data}

The case of dengue in tropic country every year becomes a problem for the government. The study area in this study is several cities which are high DHF cases each year; the data used are data on the number of dengue cases in Makassar city. In addition, there are assumptions for parameter and initial values are simulation data for free disease and endemic status.

\subsection{Methods}

The SIRI model in this study is a modification of the SIR model. Analysis of the SIRI model uses the Lyapunov function method [9], and then the data used in the simulation are assuming data to show two possible dengue statuses are disease free and endemic status. 
The simulation of the model also using the number of dengue cases data in Makassar city by the Department of Health in South Sulawesi. Simulation models using Maple 18 to predict the number of dengue cases in Makassar city in the following month.

\section{Results and Discussion}

\subsection{SIRI Model for Dengue Fever Disease}

Changes that occur in each human population and vector population of SIRI model for the transmission of dengue disease can be interpreted in Figure 1.

According to Figure 1, SIRI model for dengue fever transmission can be interpreted in system (1):

$$
\left\{\begin{array}{l}
\frac{d S_{h}}{d t}=\mu_{h} N_{h}-\left(\frac{\beta_{h} b}{N_{h}} I_{v}+\mu_{h}\right) S_{h} \\
\frac{d I_{h}}{d t}=\frac{\beta_{h} b}{N_{h}} I_{v} S_{h}+\left(\frac{\alpha_{h} I_{v} b}{N_{h}}\right) R_{h}-\left(\mu_{h}+\gamma_{h}\right) I_{h} \\
\frac{d R_{h}}{d t}=\gamma_{h} I_{h}-\left(\mu_{h}+\frac{\alpha_{h} I_{v} b}{N_{h}}\right) R_{h} \\
\frac{d S_{v}}{d t}=\mu_{v} N_{v}-\frac{\beta_{v} b}{N_{h}} I_{h} S_{v}-\mu_{v} S_{v} \\
\frac{d I_{v}}{d t}=\frac{\beta_{v} b}{N_{h}} I_{h} S_{v}-\mu_{v} I_{v}
\end{array}\right.
$$

Simplify system (1) with $S_{h}=\frac{S_{h}}{N_{h}} ; I_{h}=\frac{I_{h}}{N_{h}} ; R_{h}=\frac{R_{h}}{N_{h}} ; S_{v}=\frac{S_{v}}{N_{v}} ;$ and $I_{v}=\frac{I_{v}}{N_{v}}$

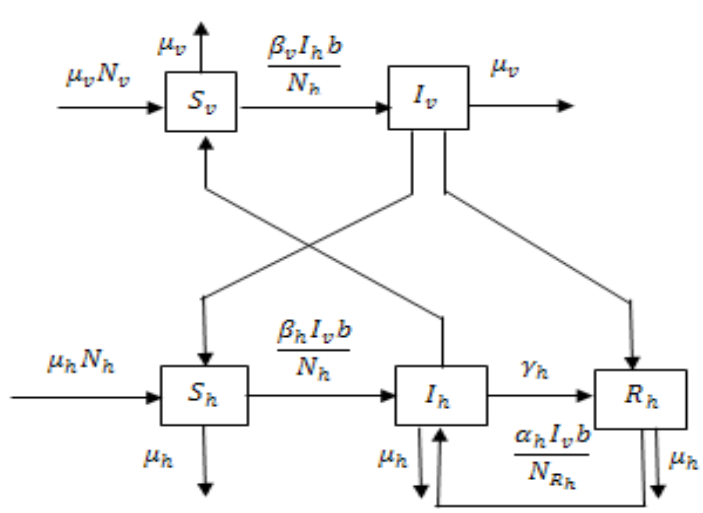

FIGURE 1. SIRI model for dengue fever transmission. 


$$
\left\{\begin{array}{l}
\frac{d S_{h}}{d t}=\mu_{h}-\eta I_{v} S_{h}-\mu_{h} S_{h} \\
\frac{d I_{h}}{d t}=\eta I_{v} S_{h}+\frac{\alpha_{h} R_{h} I_{v} \eta}{\beta_{h}}-\left(\mu_{h}+\gamma_{h}\right) I_{h} \\
\frac{d R_{h}}{d t}=\gamma_{h} I_{h}-\mu_{h} R_{h}-\frac{\alpha_{h} R_{h} I_{v} \eta}{\beta_{h}} \\
\frac{d S_{v}}{d t}=\mu_{v}-\beta_{v} b I_{h} S_{v}-\mu_{v} S_{v} \\
\frac{d I_{v}}{d t}=\beta_{v} b I_{h} S_{v}-\mu_{v} I_{v}
\end{array}\right.
$$

where $\eta=\frac{b \beta_{h} N_{v}}{N_{h}}$.

The system (2) is a SIRI model for dengue fever trasmission.

\subsection{Global Stability Analysis of SIRI Model for Dengue Fever Disease}

\subsubsection{Stability Analysis of SIRI Model for Dengue Fever Disease}

The stability of the model towards the balance points obtained is determined by eigen values $\lambda$ as in Table 1 .

The types of stability of the system depend on eigen values with the type of stability shown in Table 1 . The eigen value is obtained by determining the matrix of system (2) then substituting the fixed point in the case of disease-free, by Maple 18, the eigen value obtained for the system of equation (2) by Jacobian matrix of system (2) is:

$$
J^{*}=\left[\begin{array}{ccccc}
-\mu_{h} & 0 & 0 & 0 & -\eta \\
0 & -\left(\mu_{h}+\gamma_{h}\right) & 0 & 0 & \eta \\
0 & \gamma_{h} & -\mu_{h} & 0 & 0 \\
0 & -\beta_{v} b & 0 & -\mu_{v} & 0 \\
0 & \beta_{v} b & 0 & 0 & -\mu_{v}
\end{array}\right]
$$

then $\operatorname{det}\left(\lambda I-J^{*}\right)=0 \mathrm{~mm}$

TABLE 1. Stability types for equilibrium points

Eigen values $\lambda_{i} ; \mathrm{i}=1,2, \ldots$
Real and all positive
Real and all negative
Real and different sign
Complex, original imaginer
Complex, $\lambda \pm \eta i, \quad \lambda>0$
Complex, $\lambda \pm \eta i, \quad \lambda<0$

Eigen values $\lambda_{i} ; \mathrm{i}=1,2, \ldots$

Real and all positive

Real and all negative

Real and different sign

Complex, original imaginer

Complex, $\lambda \pm \eta i, \quad \lambda<0$
Stability

Node unstable

Asymptotic stable nod

SaddlePoint

Center

Focus and unstable

Focus and asymptotic stable 


$$
\left(\lambda+\mu_{h}\right)\left(\lambda+\mu_{h}\right)\left(\lambda+\mu_{v}\right)\left(\lambda^{2}+\left(\mu_{h}+\mu_{v}+\gamma_{h}\right) \lambda+\left(\mu_{v}\left(\mu_{h}+\gamma_{h}\right)-\eta \beta_{v} b\right)=0\right.
$$

The eigen value of model is:

$$
\begin{gathered}
\lambda_{1}=\frac{-\left(\mu_{h}+\mu_{v}+\gamma_{h}\right)+\sqrt{\left(\mu_{h}\right)^{2}-2 \mu_{h} \mu_{v}+2 \mu_{h} \gamma_{h}+\left(\mu_{v}\right)^{2}+5 \gamma_{h} \mu_{v}+\left(\gamma_{h}\right)^{2}+4 \eta \beta_{v} b}}{2} \\
\lambda_{2}=\frac{-\left(\mu_{h}+\mu_{v}+\gamma_{h}\right)+\sqrt{\left(\mu_{h}\right)^{2}-2 \mu_{h} \mu_{v}+2 \mu_{h} \gamma_{h}+\left(\mu_{v}\right)^{2}+5 \gamma_{h} \mu_{v}+\left(\gamma_{h}\right)^{2}+4 \eta \beta_{v} b}}{2} \\
\lambda_{3}=-\mu_{h} \\
\lambda_{4}=-\mu_{v} \\
\lambda_{5}=-\left(\mu_{h}+\gamma_{h}\right)
\end{gathered}
$$

\subsubsection{Basic Reproduction Number}

The basic reproduction number is the expected value of the number of infections per time. This infection occurs in a suspectible population infected by an infected individual.

According to the eigen equation (3) of system (2), the basic reproduction number of the SIRI model for dengue fever transmission is:

$$
R_{0}=\frac{\eta \beta_{v} b}{\mu_{v}\left(\mu_{h}+\gamma_{h}\right)}
$$

\subsection{Simulation of SIRI Model for Dengue Fever Transmission in Free Disease Case}

The SIRI model simulation by using Maple with initial and parameter values based on the assumptions is presented in Table 2, with basic reproduction number values obtained based on equation (9), namely:

$$
R_{0}=\frac{\eta \beta_{v} b}{\mu_{v}\left(\mu_{h}+\gamma_{h}\right)}
$$

To determine the critical point, with the substitution of parameter values for diseasefree cases in Table 2, system equation (2) is equaled to zero, then the following system of equations (1) is obtained:

$$
\begin{cases}0.0146-0.0245 I_{v} S_{h}-0.0146 S_{h} & =0 \\ 0.0245 S_{h}+0.084 I_{v} R_{h}-0.3266 I_{h} & =0 \\ 0.312 I_{h}-\left(0.0146+0.084 I_{v}\right) R_{h} & =0 \\ 0.043-0.21 I_{h} S_{v}-0.043 S_{v} & =0 \\ 0.21 I_{h} S_{v}-0.043 I_{v} & =0\end{cases}
$$


TABLE 2. Initial and parameter values of the SIRI model for free disease case

\begin{tabular}{lllc}
\hline Parameter & Values $\mathbf{R}_{\mathbf{0}} \leq \mathbf{1}$ & Variable & Initial value \\
\hline$\beta_{h}$ & 0.006125 & $N_{h}(0)$ & 2500 \\
$\mu_{h}$ & 0.0146 & $S_{h}(0)$ & 1300 \\
$b$ & 10 & $I_{h}(0)$ & 800 \\
$\alpha_{h}$ & 0.021 & $R_{h}(0)$ & 400 \\
$\gamma_{h}$ & 0.312 & $N_{v}(0)$ & 1000 \\
$\mu_{v}$ & 0.043 & $S_{v}(0)$ & 700 \\
$\beta_{v}$ & 0.021 & $I_{v}(0)$ & 300 \\
\hline
\end{tabular}

The system (10) provides equilibrium points of the SIRI model for free disease model are:

$$
\left(S_{h}, I_{h}, R_{h}, S_{v}, I_{v}\right)=(0.3085 ; 0.0822 ; 0.7737 ; 0.6895 ; 0.3065)
$$

The equilibrium points explain that the number of suspected human populations is 3085 people, infected with dengue fever as many as 822 people, the number of people recovering is 7737 people out of the total 10,000 human population.

Eigen values with parameter in Table 2 of SIRI model for DHF transmission are:

$$
\lambda_{1}=-0.0146, \lambda_{2}=-0.0146, \lambda_{3}=-0.043, \lambda_{4}=-0.5682 \text { and } \lambda_{5}=-0.0667
$$

The values obtained are real and negative, so based on Table 1, then this type of stability at this equilibrium point is asymptotic stable. While the basic reproduction number for dengue fever transmission is, this means that infected individuals cannot transmit to other individuals that cause the disease to disappear from the population or the stage of DHF is disease free disease case.

\subsubsection{Simulation Results of SIRI Model for Dengue Fever Transmission in Free Disease Case}

The simulation results of the SIRI model of DHF transmission for disease-free cases were obtained by Maple software with the initial values and parameters as in Table 2 shown in Figure 2. The $x$-axis and $y$-axis coordinates represent the time (month) and number of cases for each variables $S_{h}(t), I_{h}(t), R_{h}(t), S_{v}(t)$, and $I_{v}(t)$.

Based on Figure 2, it can be concluded that DHF number cases for infected populations will continue to decline and the disease will disappear in the 12th month, this is caused by the value of $R_{0}<1$, which means that infected individuals cannot transmit the disease to other individuals. Decreasing the number of infected populations also causes suspect populations to continue to increase.

\subsection{Simulation of SIRI Model for Dengue Fever Transmission in Endemic Case}

The SIRI model simulation by using Maple with initial and parameter values based on the assumptions is presented in Table 3. 


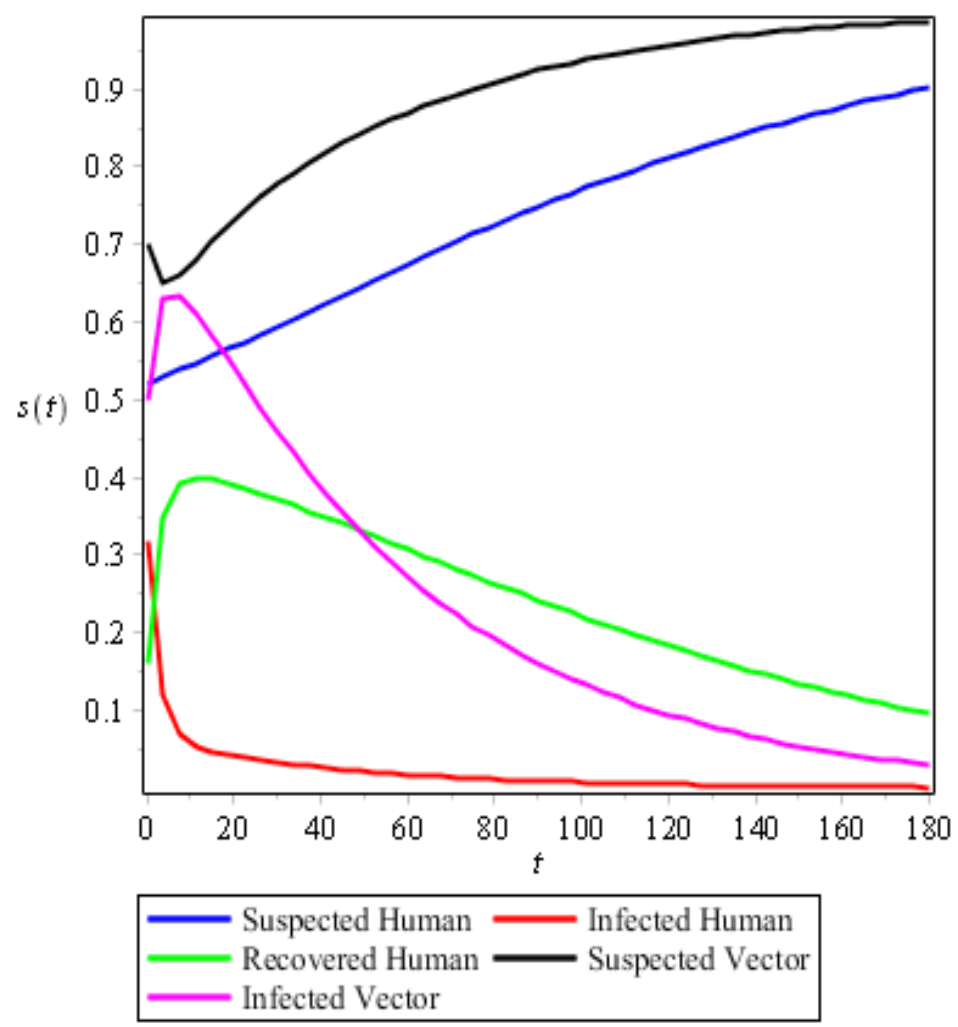

FIGURE 2. Prediction of suspected, infeted, recovered number for free disease case of DHF.

To determine equilibrium point, with the substitution of parameter values for endemic cases in Table 3, system equation (2) is equal to zero, then the following system of equations (11) is obtained:

$$
\begin{cases}0.0236-0.312 I_{v} S_{h}-0.0236 S_{h} & =0 \\ 0.312 S_{h}+2.22 I_{v} R_{h}-0.1796 I_{h} & =0 \\ 0.156 I_{h}-\left(0.0236+2.22 I_{v}\right) R_{h} & =0 \\ 0.026-0.6765 I_{h} S_{v}-0.026 S_{v} & =0 \\ 0.6765 I_{h} S_{v}-0.026 I_{v} & =0\end{cases}
$$

The equation (11) system provides equlibrium points for SIRI endemic model are:

$$
\left(S_{h}, I_{h}, R_{h}, S_{v}, I_{v}\right)=(0.0447 ; 0.7591 ; 0.1962 ; 0.00051 ; 0.9944)
$$

These equilibrium points shown that the number of suspected human populations is 447 people, infected with dengue is 7591 people and the number of recovered is 1962 people out of the total 10,000 human population.

Eigen values with parameter in Table 3 of SIRI model for DHF transmission are: 


$$
\lambda_{1}=-0.0236, \lambda_{2}=-0.0236, \lambda_{3}=-0.026, \lambda_{4}=-4.9155 \text { and } \lambda_{5}=-4.2887
$$

The values $\lambda$ obtained are real and negative, so based on Table 1 , then this type of stability at this equilibrium point is asymptotic stable. While the basic reproduction number for dengue fever disease is, this means that someone who is infected with DHF will transmit to 31 other people or the stage of DHF is endemic case.

\subsubsection{Simulation Results of SIRI Model for DHF in Endemic Case}

The simulation results of the SIRI model of DHF transmission for endemic cases were obtained by MAPLE software with the initial values and parameters as in Table 3 . The $x$-axis and $y$-axis coordinates represent the time (month) and number of cases for each variables $\mathrm{S}_{\mathrm{h}}(\mathrm{t}), \mathrm{I}_{\mathrm{h}}(\mathrm{t}), \mathrm{R}_{\mathrm{h}}(\mathrm{t}), \mathrm{S}_{\mathrm{v}}(\mathrm{t})$, and $\mathrm{I}_{\mathrm{v}}(\mathrm{t})$.

Based on Figure 3, it can be concluded that the number of infected cases will increase for the next months because the stage of this cases is endemic (basic reproduction number, $R_{0}$ $>1$ ). It accordance to the SIRI model for DHF transmission in endemic cases that dengue disease will continue to develop in the population and causing outbreaks.

\subsection{Simulation of SIRI Model for Dengue Fever Transmission in Makassar}

The SIRI model simulation using Maple with initial and parameter values based on the assumptions is presented in Table 4. To determine equilibrium point, with the substitution of parameter values for endemic cases in Table 4, system equation (2) is equal to zero, and then the following system (12) is obtained:

$$
\left\{\begin{array}{l}
0.0046-0.2925 I_{v} S_{h}-0.0046 S_{h}=0 \\
0.2925 I_{v} S_{h}+0.000042 I_{v} R_{h}-0.33433 I_{h}=0 \\
0.32833 I_{h}-\left(0.0046+0.000042 I_{v}\right) R_{h}=0 \\
0.00323-0.0156 I_{h} S_{v}-0.00323 S_{v}=0 \\
0.0156 I_{h} S_{v}-0.00323 I_{v}=0
\end{array}\right.
$$

The system (12) provides equlibrium points for SIRI dengue fever transmission in Makassar city, South Sulawesi, Indonesia are:

TABLE 3. Initial values and parameters of SIRI model for endemic case of DHF

\begin{tabular}{llll}
\hline Parameter & Values $\mathbf{R}_{\mathbf{0}} \leq \mathbf{1}$ & Variable & Initial value \\
\hline$\beta_{h}$ & 0.0052 & $N_{h}(0)$ & 2500 \\
$\mu_{h}$ & 0.0236 & $S_{h}(0)$ & 1300 \\
$b$ & 150 & $I_{h}(0)$ & 800 \\
$\alpha_{h}$ & 0.037 & $R_{h}(0)$ & 400 \\
$\gamma_{h}$ & 0.156 & $N_{v}(0)$ & 1000 \\
$\mu_{v}$ & 0.026 & $S_{v}(0)$ & 700 \\
$\beta_{v}$ & 0.451 & $I_{v}(0)$ & 300 \\
\hline
\end{tabular}




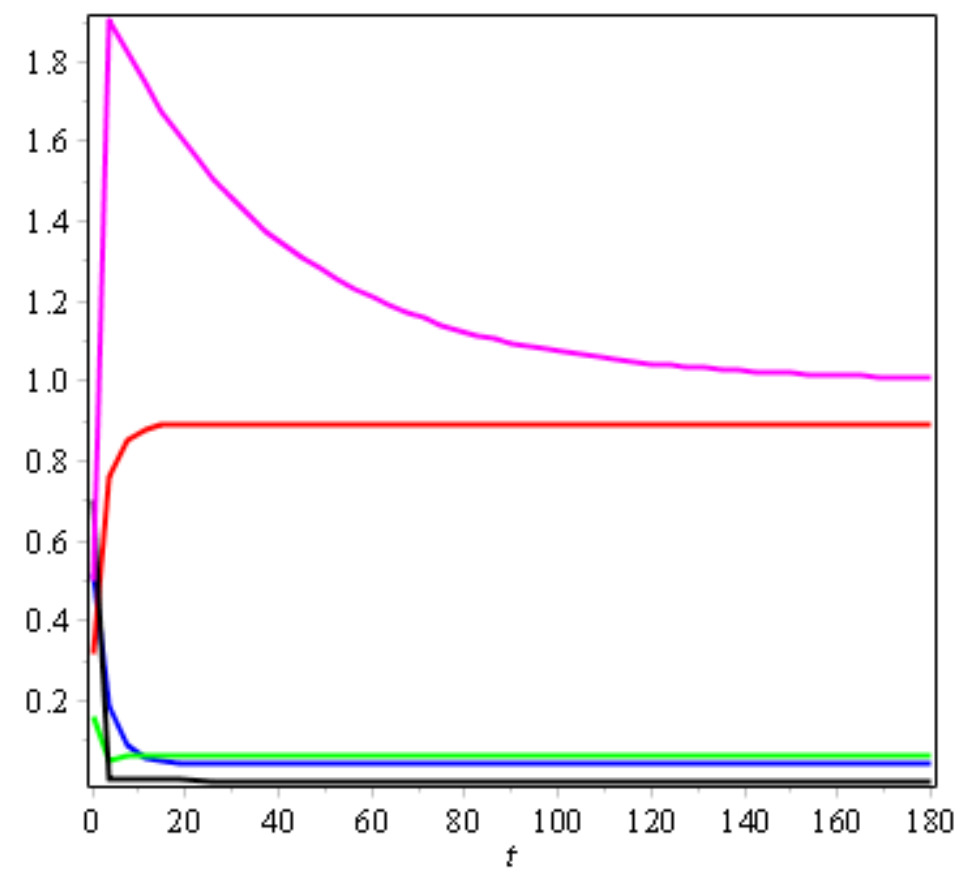

Suspected Human

Infected Human

Recovered Human

Suspected Vector

Infected Vector

FIGURE 3. Prediction of suspected, infeted, recovered number for endemiccase of DHF.

TABLE 4. Initial and parameter values of SIRI model for dengue fever in Makassar

\begin{tabular}{lll}
\hline Variable & Initial value & Source \\
\hline$N_{h}(0)$ & 1663479 & {$[17]$} \\
$S_{h}(0)$ & 1663212 & {$[17]$} \\
$I_{h}(0)$ & 135 & {$[17]$} \\
$R_{h}(0)$ & 132 & {$[17]$} \\
$N_{v}(0)$ & 1000 & {$[16]$} \\
$S_{v}(0)$ & 350 & {$[16]$} \\
$I_{v}(0)$ & 56 & {$[16]$} \\
\hline Parameter & Values $\mathbf{R}_{\mathbf{0}} \leq \mathbf{1}$ & Source \\
\hline$\beta_{h}$ & 0.20124 & KKRI [16] \\
$\mu_{h}$ & 0.0046 & KKRI [16] \\
$b$ & 150 & Assumption \\
$\alpha_{h}$ & 0.000045 & KKRI [16] \\
$\gamma_{h}$ & 0.3288 & KKRI [16] \\
$\mu_{v}$ & 0.00323 & KKRI [16] \\
$\beta_{v}$ & 0.0001 & KKRI [16] \\
\hline
\end{tabular}




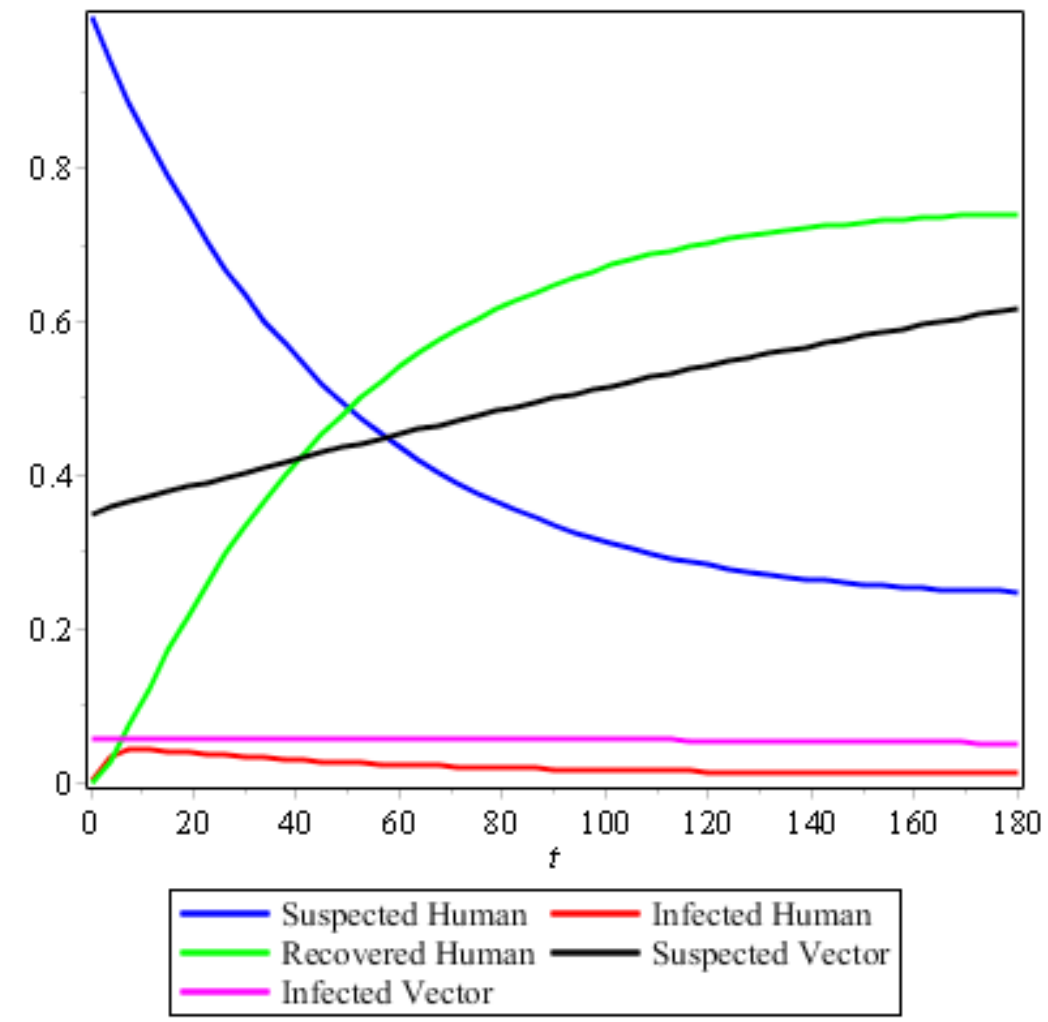

FIGURE 4. Prediction of suspected, infeted, recovered number for DHF in Makassar.

$$
\left(S_{h}, I_{h}, R_{h}, S_{v}, I_{v}\right)=(0.4209 ; 0.0046 ; 0.5745 ; 0.1116 ; 0.9784)
$$

These equilibrium points shown that the number of suspected human populations is 420,900 people, infected with dengue is 46,000 people and the number of recovered is 574,500 people out of the total 1,000,000 human population.

Eigen values with parameter values in Table 3 for the SIRI model of DHF transmission are:

$$
\lambda_{1}=-0.0046, \lambda_{2}=-0.0046, \lambda_{3}=-0.00323, \lambda_{4}=-0.587 \text { and } \lambda_{5}=-0.0019
$$

The values $\lambda$ obtained are real and negative, so based on Table 1 , then this type of stability at this equilibrium point is asymptotic stable. While the basic reproduction number for dengue fever found $R_{0}=0.0272<1$, this means that infected individuals cannot transmit to other individuals in Makassar city that cause the disease to disappear from the population or the stage of DHF in Makassar city, South Sulawesi Indonesia is free disease case.

\subsubsection{Simulation Results of SIRI Model for Dengue Fever Transmission in Makassar}

The simulation results of the SIRI model of DHF transmission in Makassar city were obtained by Maple software with the initial values and parameters as in Table 4 shown 
in Figure 4. The $x$-axis and $y$-axis coordinates represent the time (month) and number of cases for each variables $S_{h}(t), I_{h}(t), R_{h}(t), S_{v}(t)$, and $I_{v}(t)$. Based on Figure 4 , it can be concluded that DHF number cases for infected populations will continue to decline and the disease will disappear in the 14 th month, this is caused by the value of $R_{0}<1$, which means that infected individuals cannot transmit the disease to other individuals in Makassar city that cause the disease to disappear from the population or the stage of DHF in Makassar city is free disease case.

\section{Conclusion}

The SIRI model for dengue fever transmission have been found, which is a modification of SIR model; analysis of the SIRI model is shown that the equation system in the model is asymptotic stable which means that dengue transmission still continues at a certain time in Makassar city, Indonesia. Whereas, the SIRI simulation model uses Maple, giving an illustration of the number of dengue patients in the following month, both for disease-free and endemic cases. In Makassar City, the prediction of dengue fever will reach its peak in the 14th month, but Makassar City is not endemic for DHF, however early anticipation needs to be done because the cycle of DHF sufferers in Makassar city, Indonesia is very fast from the three-years cycle become fourteen months.

\section{Data Availability}

The data used to finding support of this study are available from the corresponding author upon request.

\section{Conflicts of Interest}

The authors declare that they have no conflicts of interest.

\section{Acknowledgment}

We would like thank to DIKTI No:124/UN36.9/PL/2019 for the financial supports.

\section{References}

1. Dengue: dengue fact sheet. http://www.searo.who.int/entity/vector_borne_tropical_diseases/ data/data_factsheet/en/\#targetText=Dengue\%20Fact\%20Sheet\&targetText=As\%20per\%20 the\%20World\%20Health,resulting\%20in\%2020\%20000\%20deaths. Date accessed: 2016.

2. Favier C. Early determination of the reproductive number for vector-borne disease: the case of dengue in Brazil. Tropical Medicine \& International Health. 2006; 11(3), 332-340.

3. Guihua L, Zhen J. Global stability of an SEI epidemic model. Chaos, Solitons \& Fractals. 2009; 21(4), 925-931. 
4. Nur W. SIR Model analysis for transmission of dengue fever disease with climate factors using Lyapunov function. Journal of Physics: Conference Series. 2018; 1028, 1-8.

5. Poerwanto B. A matlab code to compute prediction of survival trends in patients DHF. Journal of Physics: Conference Series. 2018; 1028, 1-8.

6. Rangkuti YM, Side S, Noorani MSM. SEIR model for transmission of dengue fever. ITB Journal of Science (Journal of Mathematical and Fundamental Sciences). 2014; 46(1), 91-105.

7. Syafruddin S, Usman M, Sahlan S, Wahidah S. A SEIR model for transmission of tuberculosis. https://aip.scitation.org/doi/10.1063/1.4980867. Date accessed: 2017.

8. Syafruddin S, Noorani MSM. Lyapunov function of SIR and SEIR model for transmission of dengue fever disease. International Journal of Simulation and Process Modelling (IJSPM). 2013; 8(2/3), 177-184.

9. Syafruddin S, Irwan, Usman M, Wahidah S. SEIR model for Hepatitis B. https://aip.scitation. org/doi/abs/10.1063/1.5002392. Date accessed: 2017.

10. Diekmann O, Heesterbeek JA, Metz JAJ. On the definition and the computation of the basic reproduction ratio $\mathrm{R}$ infectious diseases in heterogeneous populations. Journal of Mathematical Biology. 1990; 28, 365-382.

11. Side S.A Susceptible-Infected-recovered model and simulation for transmissionof tuberculosis. Advanced Science Letters. 2015; 21(2), 137-139.

12. Seem mathematics model for spatial control with the effect of vaccination in Bulukumba district. https://translate.google.com/\#view=home\&op=translate\&sl=auto\&tl=en\&text=MODEL\%20 MATEMATIKA\%20SEIR\%20UNTUK\%20KONTROL\%20CAMPAK\%20DENGAN\%20 PENGARUH\%20VAKSINASI\%20DI\%20KABUPATEN\%20BULUKUMBA. Date accessed: 2016.

13. Chaharborj SS. Behavior stability in two SIR-style models for HIV. International Journal of Math Analysis. 2010; 4(9), 427-434.

14. Thesis: stability analysis of DHF spread in Jember district using the stochastic SIR method. https://translate.google.com/\#view=home\&op=translate\&sl=auto\&tl=en\&text=Thesis \%3A\%20Analisis\%20Stabilitas\%20Penyebaran\%20DBD\%20di\%20Kabupaten\%20Jember\%20 Menggunakan\%20Metode\%20Stochastic\%20SIR. Date accessed: 2013.

15. Mathematical biology: an introduction. https://www.springer.com/gp/book/9780387952239. Date accessed: 2002.

16. Syafruddin S, Noorani MSM. A SIR model for spread of dengue fever disease (simulation for South Sulawesi Indonesia and Selangor Malaysia). World Journal of Modeling and Simulation. 2013; 9(2), 96-105.

17. Dengue fever data in South Sulawesi. https://www.kemkes.go.id/resources/download/pusdatin/ profil-kesehatan-indonesia/Data-dan-Informasi_Profil-Kesehatan-Indonesia-2018.pdf. Date accessed: 2018 